\title{
Transport of solar wind fluctuations: A two-component model
}

\author{
S. Oughton, ${ }^{1}$ W. H. Matthaeus, ${ }^{2}$ C. W. Smith, ${ }^{3}$ B. Breech,,${ }^{4,5}$ and P. A. Isenberg ${ }^{3}$ \\ Received 9 December 2010; revised 28 March 2011; accepted 4 May 2011; published 26 August 2011.
}

[1] We present a new model for the transport of solar wind fluctuations which treats them as two interacting incompressible components: quasi-two-dimensional turbulence and a wave-like piece. Quantities solved for include the energy, cross helicity, and characteristic transverse length scale of each component, plus the proton temperature. The development of the model is outlined and numerical solutions are compared with spacecraft observations. Compared to previous single-component models, this new model incorporates a more physically realistic treatment of fluctuations induced by pickup ions and yields improved agreement with observed values of the correlation length, while maintaining good observational accord with the energy, cross helicity, and temperature.

Citation: Oughton, S., W. H. Matthaeus, C. W. Smith, B. Breech, and P. A. Isenberg (2011), Transport of solar wind fluctuations: A two-component model, J. Geophys. Res., 116, A08105, doi:10.1029/2010JA016365.

\section{Introduction}

[2] Fluctuations in the velocity and magnetic fields of the solar wind play important roles in various processes in the heliosphere, including scattering of solar energetic particles [Bieber et al., 1994; Dröge et al., 2010], modulation of galactic cosmic rays [Zank et al., 1998; Pei et al., 2010], and heating of the solar wind itself [Coleman, 1968; Matthaeus et al., 1999; Cranmer and van Ballegooijen, 2003]. The spatial transport and evolution of these fluctuations is thus a topic of some importance in heliospheric physics. Here we present such a transport model, specifically, one that treats the fluctuations as an admixture of two incompressible components: quasi-two-dimensional (quasi-2-D) turbulence and (Alfvén) wave-like fluctuations [Matthaeus et al., 1990; Tu and Marsch, 1993; Bieber et al., 1994]. For each component, transport equations for its energy, cross helicity, and correlation length are developed, and numerical solutions are shown to compare favorably with observations.

[3] Early transport models assumed that (parallelpropagating) Alfvén waves were the dominant fluctuation type and used WKB-based approaches [Parker, 1965; Belcher, 1971], eventually supported by evidence for Alfvén waves [e.g., Belcher and Davis, 1971]. On the other hand, observations also suggested that turbulence was present and may have contributed to heating [e.g., Coleman, 1968]. Subsequent observational studies indicated that at magne-

\footnotetext{
${ }^{1}$ Department of Mathematics, University of Waikato, Hamilton, New Zealand.

${ }^{2}$ Bartol Research Institute and Department of Physics and Astronomy, University of Delaware, Newark, Delaware, USA.

${ }^{3}$ Institute for the Study of Earth, Oceans, and Space, University of New Hampshire, Durham, New Hampshire, USA.

${ }^{4}$ NASA Goddard Space Flight Center, Greenbelt, Maryland, USA.

${ }^{5}$ Now at U. S. Army Research Laboratory, Aberdeen Proving Grounds, Aberdeen, Maryland, USA.

Copyright 2011 by the American Geophysical Union. 0148-0227/11/2010JA016365
}

tohydrodynamic (MHD) scales there are at least two distinct types of fluctuation and that the wave-like energy may be a minor component [Matthaeus et al., 1990; Bieber et al., 1996; Smith, 2003; Milano et al., 2004; Dasso et al., 2005; Horbury et al., 2005, 2008; Podesta, 2009; Osman and Horbury, 2009; Narita et al., 2010]. This encouraged development of more complete transport theories for the energy-containing range quantities [e.g., Tu and Marsch, 1993; Matthaeus et al., 1994, 1996, 1999, 2004; Zank et al., 1996; Smith et al., 2001, 2006; Isenberg et al., 2003, 2010a; Isenberg, 2005; Breech et al., 2005, 2008; Yokoi and Hamba, 2007; Usmanov and Goldstein, 2010; Ng et al., 2010], in which turbulence properties are built in, contrasting with WKB theory, in which the waves are noninteracting at leading order. The agreement between observations made from 0.3 to $80 \mathrm{AU}$ and numerical solutions to these models has also improved, with reasonable accord achieved for the radial evolution of the energy, cross helicity $\left(H_{c}\right)$, and correlation length of the fluctuations and also for the proton temperature. Turbulence transport models have also been used in scattering theory to obtain cosmic ray mean-free paths in various heliospheric positions and conditions [Florinski et al., 2003; Florinski and Pogorelov, 2009; Pei et al., 2010]. In this way, energetic particle observations and models provide, in effect, an additional type of observational constraint on transport modeling. In general, as additional physical effects have been incorporated, this family of transport models has been able to better account for observational constraints.

[4] Nonetheless, various shortcomings in the models remain to be addressed. For example, driving of the fluctuations enters in at least two separate ways: Stream shear injects energy into low-frequency fluctuations, whereas pickup ions couple to fluctuations with much higher frequencies and shorter length scales. Accounting consistently for both types of driving in models with only a single type of fluctuation is clearly problematic. In a two-component model, however, the physics of the driving processes can be cleanly assigned 
to the appropriate component, and this is an important advantage of the model described herein.

[5] Note that the wave-like fluctuations will typically not strictly obey a dispersion relation for linearized wave modes, since there are nonlinear effects. The moniker is intended to indicate that although there are wave aspects (such as propagation) to these fluctuations, there are also other important features of their dynamics. It is perhaps more helpful to think of the quasi-2-D and wave-like components as being the low-frequency and high-frequency contributions, respectively, to the incompressible fluctuations.

[6] The remainder of this paper is structured as follows. In section 2 we sketch the model's derivation and then, in section 3, compare numerical solutions with observational data. A discussion section follows, and the paper closes with an appendix that presents the incompressible, homogeneous two-component phenomenology for the $H_{c} \neq 0$ case. Our focus herein is on development of a model for the transport of quantities at the energy-containing scales. For discussion of solar wind transport models for spectra or correlation functions, see, e.g., Tu and Marsch [1993, 1995]. An $H_{c}=0$ version of the present model was also recently presented by Isenberg et al. [2010b].

\section{Model}

[7] Here we outline the derivation of the two-component transport model. Since much of this development parallels that of the single-component models, we refer readers desiring more details to a study by Breech et al. [2008], which contains a thorough account of the derivation of (singlecomponent) transport equations for incompressible solar wind fluctuations. It is, however, helpful to list some key approximations used in developing the model: (1) scale separation between the fluctuations and the background fields, (2) locally incompressible fluctuations, (3) large-scale fields specified and time steady (density $\rho$, wind speed $U$, Alfvén speed $V_{A}$ ), with $U \gg V_{A}$, (4) assumption that largescale gradients are in the radial direction, and (5) assumption that turbulent decay leads to proton heating. See the work of Breech et al. [2008] for detailed discussion of the approximations and simplifications used.

[8] There are essentially two sets of terms in the transport model: (1) terms that couple the fluctuations and the largescale fields and (2) self-interaction terms for the fluctuations. The former are primarily linear and the latter are primarily nonlinear with respect to the fluctuation energies. Fortunately, modeling of the self-interaction terms can take place without consideration of transport effects, and in section 2.1 we first recap the development of a de Kármán-Howarth phenomenology for single-component MHD and then outline our generalization to the two-component case. The full transport model is considered in section 2.2.

\subsection{Incompressible Two-Component Turbulence Model}

[9] Consider incompressible homogeneous 3-D MHD turbulence, with the governing equations expressed in terms of the Elsässer variables, $\boldsymbol{z}^{ \pm}(\boldsymbol{x}, t)=\boldsymbol{v} \pm \boldsymbol{b}$; here $\boldsymbol{b}$ is the magnetic fluctuation in Alfvén speed units. Denote the energy of the fluctuations as $Z^{2} / 2=\left\langle\boldsymbol{z}^{+} \cdot \boldsymbol{z}^{+}+\boldsymbol{z}^{-} \cdot \boldsymbol{z}^{-}\right\rangle / 4$, where angle brackets denote a Reynolds decomposition, often taken to be well approximated by an appropriate average over $\boldsymbol{x}$. Note that we develop the local turbulence model with the average mean flow set to zero, so that the turbulence is temporarily treated as homogeneous. Let $\ell$ be a characteristic length scale for the fluctuations (e.g., a correlation length). The associated eddyturnover time scale for the fluctuations is thus $\tau=\ell / Z$.

[10] The de Kármán and Howarth [1938] approach is to use the characteristic speed $(Z)$ and length scale $(\ell)$ of the energy-containing scales of the flow to construct a simple phenomenology for the evolution of $Z^{2}$ and $\ell$ by assuming that they both relax on the time scale $\tau$ :

$$
\begin{gathered}
\frac{\mathrm{d} Z^{2}}{\mathrm{~d} t}=-\alpha \frac{Z^{2}}{\tau}=-\alpha \frac{Z^{3}}{\ell}, \\
\frac{\mathrm{d} \ell}{\mathrm{d} t}=\beta \frac{\ell}{\tau}=\beta Z,
\end{gathered}
$$

with $\alpha$ and $\beta$ constants. Indeed, if we identify $Z^{2} / 2$ with just the kinetic energy, equations (1) and (2) express precisely the de Kármán and Howarth [1938] phenomenology for the Navier-Stokes equations; they give good agreement with experimental results for high enough Reynolds numbers [e.g., Sreenivasan, 1995]. Associated with this phenomenology is the conservation law $\ell Z^{2 \beta / \alpha}=$ const. Physically, $2 \beta / \alpha=1$ corresponds to decay at constant Reynolds numbers, and $2 \beta / \alpha=2$ corresponds to decay subject to constant area under the sum of the $\boldsymbol{v}$ and $\boldsymbol{b}$ correlation functions [Zank et al., 1996; Matthaeus et al., 1996].

[11] A key element of the modeling is the replacement of terms like $\left\langle z^{+} \cdot\left(z^{-} \cdot \nabla\right) z^{+}\right\rangle$, in the exact equation for $Z^{2}$, with $Z^{3} / \ell$. This involves treating $Z^{+} \approx Z$, i.e., assuming small cross helicity. Extensions that support arbitrary cross helicity and a mean magnetic field $\boldsymbol{B}_{0}$ have been proposed [Dobrowolny et al., 1980; Hossain et al., 1995; Matthaeus et al., 1994].

[12] Our objective here is to generalize this approach to encompass decomposition of the fluctuations into two precisely defined incompressible components, the quasi-2-D and wave-like contributions, each with arbitrary cross helicity. The starting point is again incompressible 3-D MHD, with a uniform magnetic field $\boldsymbol{B}_{0}$ (and associated Alfvén speed $V_{A}$ ). The zero-cross-helicity case is developed by Oughton et al. [2006] and is also sketched below. Development of the arbitrary cross helicity case is straightforward but more algebraically involved.

[13] Let the total Elsässer fluctuation(s) be written as

$$
\boldsymbol{z}^{ \pm}=\boldsymbol{q}^{ \pm}+\boldsymbol{w}^{ \pm}
$$

where $\boldsymbol{q}^{ \pm}$are the quasi-2-D (nominally low-frequency) components and $\boldsymbol{w}^{ \pm}$are the wave-like (high-frequency) components. Propagation and other features of waves may be important in the wave-like component, whereas nonlinear processes predominate for the quasi-2-D component.

[14] The $\boldsymbol{q}^{ \pm}$and $\boldsymbol{w}^{ \pm}$can be rigorously defined, within the context of the incompressible model, using Fourier decomposition and the ratio of the nonlinear time scale $\tau_{\mathrm{nl}}(\boldsymbol{k})$ and Alfvén time scale $\tau_{A}(\boldsymbol{k})=1 /\left(\boldsymbol{k} \cdot \boldsymbol{V}_{A}\right)$ for each Fourier mode [Oughton et al., 2006]. When the time scales at a specific wave vector $\boldsymbol{k}$ are such that $\tau_{\mathrm{nl}} \leq \tau_{A}$, then the fluctuations at 
Table 1. Definitions of Some Important Physical Variables for the Quasi-2-D and Wave-Like Components ${ }^{\mathrm{a}}$

\begin{tabular}{llc}
\hline \multicolumn{1}{c}{$Z$ Quantity } & \multicolumn{1}{c}{ Description } & $W$ Quantity \\
\hline$Z_{ \pm}^{2}=\left\langle\boldsymbol{q}_{ \pm} \cdot \boldsymbol{q}_{ \pm}\right\rangle$ & Elsässer “energies" & $W_{ \pm}^{2}=\left\langle\boldsymbol{w}_{ \pm} \cdot \boldsymbol{w}_{ \pm}\right\rangle$ \\
$2 Z^{2}=Z_{+}^{2}+Z_{-}^{2}$ & $\propto$ total energy & $2 W^{2}=W_{+}^{2}+W_{-}^{2}$ \\
$4 H_{c}^{Z}=Z_{+}^{2}-Z_{-}^{2}$ & cross helicity & $4 H_{c}^{W}=W_{+}^{2}-W_{-}^{2}$ \\
$\sigma_{c}=\frac{Z_{+}^{2} Z_{-}^{2}}{Z_{+}^{2}+Z_{-}^{2}}$ & normalized cross helicity & $\tilde{\sigma}_{c}=\frac{W_{+}^{2}-W_{-}^{2}}{W_{+}^{2}+W_{-}^{2}}$ \\
$\sigma_{D}=\frac{\left\langle\boldsymbol{q}_{+} \cdot \boldsymbol{q}_{-}\right\rangle}{Z^{2}}$ & normalized energy difference & $\tilde{\sigma}_{D}=\frac{\left\langle\boldsymbol{w}_{+} \cdot \boldsymbol{w}_{-}\right\rangle}{W^{2}}$ \\
\hline
\end{tabular}

${ }^{a}$ The normalized energy difference is equal to the (kinetic - magnetic)/ (kinetic + magnetic) energy in the component.

that $\boldsymbol{k}$ belong to the quasi-2-D population, since the nonlinear dynamics is fast compared to the wave dynamics. This is more likely to occur when $\boldsymbol{k}$ is quasi-perpendicular to $\boldsymbol{V}_{A}$, which motivates the name for the component. When $\tau_{\mathrm{nl}}(\boldsymbol{k}) \geq$ $\tau_{A}(\boldsymbol{k})$, the fluctuations are grouped with the wave-like population. Note that the components are mutually exclusive. An important property of the components is that they obey orthogonality relations such as $\left\langle\boldsymbol{q}^{+} \cdot \boldsymbol{w}^{+}\right\rangle=0$. However, mixed inner product terms such as $\left\langle\boldsymbol{q}^{+} \cdot \boldsymbol{w}^{-}\right\rangle \neq 0$ do not vanish, in general. A proof of the orthogonality is outlined at the end of Appendix A.

[15] To develop the phenomenology, first substitute the decomposition (equation (3)) into the equations for 3-D incompressible MHD with a uniform magnetic field. Next, take dot products of the MHD equations with $\boldsymbol{q}^{ \pm}$and $\boldsymbol{w}^{ \pm}$ (separately), and average them over space. This process yields equations for the evolution of the Elsässer energies for each component, e.g., $Z_{+}^{2}$ and $W_{+}^{2}$ (see Table 1). These equations contain terms like $\left\langle\boldsymbol{w}^{+} \cdot\left(\boldsymbol{z}^{-} \cdot \nabla\right) \boldsymbol{w}^{+}\right\rangle$, which are to be modeled. A naive modeling is $W_{+}^{2} Z_{-} / \lambda$, where $\lambda$ is the characteristic length scale of the wave-like component. In fact, a multiplicative factor should be included to account for the shortening of the triple correlation time scale (see equation (A4)) associated with the wave-like propagation effects for this nonlinear term [Kraichnan, 1965; Grappin et al., 1982, 1983; Matthaeus and Zhou, 1989; Zhou et al., 2004]. Generically, these factors are $\Gamma=\left(1+\tau_{\mathrm{nl}} / \tau_{A}\right)^{-1}<1$, so that for this example term our modeling yields $\Gamma_{z}^{z w} W_{+}^{2} Z_{-} / \lambda$. Using this modeling approach for all the nonlinear terms yields the energy-like equations for the phenomenology (see Appendix A).

[16] The physical features of the two-component phenomenology can be appreciated by considering the simpler zero-cross-helicity version, which we now address. We denote the energy in the quasi-2-D fluctuations as $Z^{2} / 2$ and that in the wave-like component as $W^{2} / 2$, with respective perpendicular (relative to $\boldsymbol{B}_{0}$ ) length scales $\ell$ and $\lambda$. The zerocross-helicity and strong $V_{A}$ version of the phenomenology has [Oughton et al., 2006]

$$
\begin{gathered}
\frac{\mathrm{d} Z^{2}}{\mathrm{~d} t}=-\frac{Z^{3}}{\ell}-\frac{W Z^{2}}{\ell} \frac{2}{1+Z / W}+X_{0}, \\
\frac{\mathrm{d} W^{2}}{\mathrm{~d} t}=-\frac{Z W^{2}}{\lambda} \frac{2}{1+\lambda / \ell}-\frac{2 W^{4}}{\lambda^{2}} \frac{\lambda_{\|}}{V_{A}}-X_{0} .
\end{gathered}
$$

In these equations, $\lambda_{\|}$is the characteristic parallel length scale of the wave-like piece, and $X_{0}=2 W Z\left(W^{2}+Z^{2}\right)\left(\frac{1}{\lambda^{2}}-\frac{1}{\ell^{2}}\right) \frac{\lambda_{\|}}{V_{A}}$ is the "exchange" term between the two components, which can be positive or negative. The other terms on the right-hand side (RHS) are the decay rates associated with the $Z-Z, W-Z$, and $W-W$ interactions and energy cascades. Terms with a $Z$ in the numerator are due to resonant interactions between the $Z$ component and either itself or the $W$ component. Since $V_{A}$ is large, the $Z$ component is localized about the 2-D plane, and thus the resonant terms correspond to approximately perpendicular cascades; they are typically the leading-order terms in this model.

[17] The physics of each term is, naturally, of interest. In equation (4), the first term is a de Kármán and HowarthTaylor model [de Kármán and Howarth, 1938; Taylor, 1938] for the self-decay of the $Z$ component and is very similar to the RHS of equation (1). The second term represents decay of $Z$ due to $Z-W$ interactions. Similarly, in equation (5) the first term models the resonant perpendicular cascade of $W^{2}$ mediated by $Z$ [Shebalin et al., 1983; Bondeson, 1985; Grappin, 1986], sometimes referred to as weak turbulence [Galtier et al., 2000, 2002]. The term $\sim W^{4} / V_{A}$ in equation (5) models an Iroshnikov-Kraichnan-type (IK-type) decay of the fluctuations [Iroshnikov, 1963; Kraichnan, 1965]. It is the only term that is independent of $Z$. The factors $2 /(1+Z / W)$ and $2 /(1+\lambda / \ell)$ are the explicit forms for the triple correlation $\Gamma$-factors introduced above.

[18] Various limits of the model are also of interest. The large $V_{A}$ version given above as equations (4) and (5) reflects the well-known anisotropy of spectral energy transfer in incompressible MHD [Shebalin et al., 1983; Bondeson, 1985; Oughton et al., 1994; Goldreich and Sridhar, 1995], with the weak IK term being the only nonperpendicular cascade. If, in addition, $Z / W \ll 1$, that is, we have an energetically weak quasi-2-D component and a strong mean field, then the behavior corresponds to weak turbulence [Galtier et al., 2000, 2002]. If the $Z$ component is zero, or at least so small that the IK-type term dominates, then the energy decay rate has the Alfvén speed modified form $\sim W^{4} / V_{A}$ introduced by Dobrowolny et al. [1980]. This condition is often viewed as leading to an IK-type inertial range [e.g., $\mathrm{Ng}$ et al., 2010]. In the general model derived by Oughton et al. [2006], the strength of $V_{A}$ is arbitrary. Under conditions where $V_{A}$ is small and $Z / W \gg 1$, the quasi-2-D region expands to occupy more and more of Fourier space, with the wave-like component becoming negligible. The phenomenology then effectively reduces to the single-component model of equations (1) and (2), which would normally be associated with an underlying Kolmogorov-type inertial range [Kolmogorov, 1941].

[19] As noted above, generalizing this homogeneous twocomponent model so that it supports nonzero cross helicities, as are often observed in the solar wind, is straightforward. The equations are given in Appendix A, where it can be seen that the structure of the equations is unchanged. The important point to note is that cross helicity weakens the nonlinear interactions so that the modeled terms now each have a multiplicative (cross-helicity-dependent) attenuation factor. These attenuation factors are denoted by $f, f^{\prime}$, etc.; they approach zero or unity in appropriate limits for the cross helicities. See Matthaeus et al. [2004] for discussion of these attenuation factors in the context of a single-component transport model.

[20] To close the model, one requires equations for the perpendicular length scales $\ell$ and $\lambda$ and the parallel length scale of the wave-like component, $\lambda_{\|}$. Such equations are 
analogs of equation (2). The versions for the $H_{c}=0$ case are given by Oughton et al. [2006], and their $H_{c} \neq 0$ extensions are stated in Appendix A.

[21] Finally in this section, we comment briefly on the suitability of using incompressible MHD to model solar wind fluctuations. Observationally, density fluctuations are frequently $\lesssim 10 \%$ [Roberts et al., 1987a], so it seems reasonable to neglect magnetosonic activity at this level. Results from nearly incompressible theory [Zank and Matthaeus, 1992a, 1992b, 1993] indicate that 3-D incompressible MHD is a leading-order description when the plasma beta is large $(>1)$. Note that this situation can still have a sizable mean magnetic field, and thus a Fourier mode can have an Alfvén time scale that is short or long compared to the nonlinear time of the mode, depending on both the parallel wavenumber and the spectral amplitude. When the plasma beta is less than unity, the leading-order description is incompressible 2-D MHD, with "high"-frequency (shear) Alfvén waves entering at higher order. In either case, modeling the system as we do herein, i.e., using incompressible quasi-2-D and incompressible wave-like components, is consistent with the nearly incompressible results.

\subsection{Transport Model}

[22] As is well known, solar wind fluctuations evolve due to a handful of important effects, including wind expansion and advection, stream shear driving, nonlinear interactions (turbulent cascades), and, in the outer heliosphere, pickup ion driving. Assuming that there is scale separation between the fluctuations and the large-scale fields, transport equations for general incompressible fluctuations can be readily derived [Zhou and Matthaeus, 1990; Zank et al., 1996; Matthaeus et al., 1996; Tu and Marsch, 1993]. A comprehensive derivation and discussion of the approximations used is given by Breech et al. [2008].

[23] Here we employ the same approach, except that from the outset the fluctuations are decomposed into two precisely defined incompressible components: quasi-2-D turbulence and a complementary wave-like component. More specifically, starting from the Elsässer form for the MHD equations (with large-scale velocity and magnetic fields included), we express the fluctuations as

$$
\boldsymbol{z}^{ \pm}(r, \boldsymbol{x})=\boldsymbol{q}^{ \pm}+\boldsymbol{w}^{ \pm}
$$

where $\boldsymbol{q}^{ \pm}$and $\boldsymbol{w}^{ \pm}$are the quasi-2-D and wave-like components; both quantities are functions of the (large-scale) heliocentric radius $r$ and the small-scale displacements $\boldsymbol{x}$ from each $r$. For each component, Table 1 collects the definitions of the major energy-related quantities that appear in the transport model.

[24] Our aim is to derive transport equations for the energies and length scales (and related quantities) characterizing the fluctuations. Taking the dot product of the MHD equations with $\boldsymbol{q}^{ \pm}$and (separately) $\boldsymbol{w}^{ \pm}$, averaging over the small scales, and making use of the orthogonality properties mentioned in section 2.1 eventually gives transport equations for the Elsässer energies of each component. Adding and subtracting these produces equations for the total energy of each component and the cross helicity of each component.
[25] The next step is to model the nonlinear terms. This is done using the nonzero-cross-helicity versions of equations (4) and (5) (see Appendix A). Finally, we incorporate models for the driving effects of large-scale shear and pickup ions [e.g., Breech et al., 2008]. Specializing to steady state yields the transport equations for the energies and (normalized) cross helicities:

$$
\begin{aligned}
\frac{\mathrm{d} Z^{2}}{\mathrm{~d} r}= & -\left[1+M \sigma_{D}-C_{\mathrm{sh}}^{Z}\right] \frac{Z^{2}}{r}-\frac{\alpha f}{U} \frac{Z^{3}}{\ell} \\
& -\frac{2 \alpha f_{z w}^{+}}{U} \frac{W Z^{2}}{\ell} \frac{1}{1+Z / W}+\frac{2 \alpha X^{+}}{U} \\
\frac{\mathrm{d} W^{2}}{\mathrm{~d} r}= & -\left[1+M \tilde{\sigma}_{D}-C_{\mathrm{sh}}^{W}\right] \frac{W^{2}}{r}-\frac{\tilde{\alpha} \tilde{f}}{U} \frac{Z W^{2}}{\lambda} \frac{2}{1+\lambda / \ell} \\
& -\frac{2 \tilde{\alpha}\left(1-\tilde{\sigma}_{c}^{2}\right)}{U} \frac{W^{4} \lambda_{\|}}{\lambda^{2} V_{A}}-\frac{2 \alpha X^{+}}{U}+\frac{\dot{E}_{\mathrm{PI}}}{U} \\
\frac{\mathrm{d} \sigma_{c}}{\mathrm{~d} r}= & \frac{\alpha}{U}\left[\frac{f^{\prime} Z}{\ell}+\frac{f_{z w}^{\prime} W}{\ell} \frac{2}{1+Z / W}-\frac{2\left(\sigma_{c} X^{+}-X^{-}\right)}{Z^{2}}\right] \\
& -\left[\frac{C_{\mathrm{sh}}^{Z}-M \sigma_{D}}{r}\right] \sigma_{c}, \\
\frac{\mathrm{d} \tilde{\sigma}_{c}}{\mathrm{~d} r}= & \frac{2 \tilde{\alpha}}{U}\left[\frac{\tilde{f^{\prime}}}{1+\lambda / \ell} \frac{Z}{\lambda}+\tilde{\sigma}_{c}\left(1-\tilde{\sigma}_{c}^{2}\right) \frac{W^{2} \lambda_{\|}}{\lambda^{2} V_{A}}\right]+\frac{2 \alpha\left(\tilde{\sigma}_{c} X^{+}-X^{-}\right)}{U W^{2}} \\
& -\left[\frac{C_{\mathrm{sh}}^{W}-M \tilde{\sigma}_{D}}{r}+\frac{\dot{E}_{\mathrm{PI}}}{U W^{2}}\right] \tilde{\sigma}_{c} .
\end{aligned}
$$

We denote the heliocentric distance as $r$ and the uniform radial solar wind speed as $U$, with the latter assumed to be much larger than the Alfvén speed $V_{A}(r)$, as is observed for $r \gtrsim 0.3 \mathrm{AU}$.

[26] Appendix A and key preceding references [e.g., Breech et al., 2008] clarify many of the steps leading to the above equations, although various quantities have yet to be defined here. For the quasi-2-D component, $\sigma_{c}$ is the normalized cross helicity, $\sigma_{D}$ is the normalized energy difference, and $\alpha, \beta$ are $O(1)$ constants. In general, the analogous quantity for the wave-like component is indicated by a tilde or a superscript $W$ (see Table 1). The strength of the large-scale shear driving is controlled with the constants $C_{\mathrm{sh}}^{Z}$ and $C_{\mathrm{sh}}^{W}$. The factor $M=\cos ^{2} \psi$ arises from the coupling of the fluctuations to the large-scale gradients of the large-scale fields. Here $\psi$ is the winding angle of the large-scale magnetic field, and the fluctuation amplitudes for both the $Z$ and $W$ components are assumed to be transverse to it (i.e., parallel variances are neglected). As discussed in Appendix A, the factors $f$, $f^{\prime}$, etc., that appear in the modeled nonlinear terms depend on one or both of $\sigma_{c}$ and $\tilde{\sigma}_{c}$, and they are bounded by \pm 1 . We refer to these as attenuation factors; they represent the weakening of the nonlinear terms as the cross helicity approaches extremal values. The terms modeling exchange of energy between the two components $\left(X^{ \pm}\right)$are defined in equation (A9).

[27] Energy injection due to coupling of the fluctuations with pickup ions, $\dot{E}_{\mathrm{PI}}$, is approximated as in earlier works [e.g., Williams and Zank, 1994; Williams et al., 1995; Zank et al., 1996; Matthaeus et al., 1999, 2004; Smith et al., 
2001, 2006; Isenberg et al., 2003; Isenberg, 2005; Breech et al., 2005, 2008], although as discussed below, the impact on the length scales is modeled in a much-improved fashion. Recall that only a small fraction of the ostensibly available pickup ion energy is actually transferred to the wave-like component [Isenberg et al., 2003; Isenberg, 2005]. See section 3 for the functional form of $\dot{E}_{\mathrm{PI}}$.

[28] Equations for the evolution of the length scales are obtained in the standard way [e.g., Breech et al., 2008], with the nonlinear terms approximated using the homogeneous, incompressible two-component phenomenology (see Appendix A). This yields

$$
\begin{gathered}
\frac{\mathrm{d} \ell}{\mathrm{d} r}=-\hat{C}_{\mathrm{sh}}^{Z} \frac{\ell}{r}+\frac{\beta}{U}\left[f Z+f_{z w}^{+} \frac{2 W}{1+Z / W}-\frac{2 \ell X^{+}}{Z^{2}}\right] \\
\frac{\mathrm{d} \lambda}{\mathrm{d} r}=-\hat{C}_{\mathrm{sh}}^{W} \frac{\lambda}{r}+\frac{2 \tilde{\beta}}{U}\left[\frac{\tilde{f} Z}{1+\lambda / \ell}+\left(1-\tilde{\sigma}_{c}^{2}\right) \frac{W^{2} \lambda_{\|}}{\lambda V_{A}}+\frac{\alpha \lambda X^{+}}{\tilde{\alpha} W^{2}}\right], \\
\frac{\mathrm{d} \lambda_{\|}}{\mathrm{d} r}=-\hat{C}_{\mathrm{sh}}^{W} \frac{\lambda_{\|}}{r}+2 \tilde{\alpha}\left(1-\tilde{\sigma}_{c}^{2}\right) \frac{W^{2} \lambda_{\|}}{U V_{A} \lambda^{2}} \lambda_{\|}-\left(\lambda_{\|}-\lambda_{\mathrm{res}}\right) \frac{\dot{E}_{\mathrm{PI}}}{U W^{2}} .
\end{gathered}
$$

For the solutions presented in section 3, we assume that the shear driving is at approximately the correlation scale and therefore set $\hat{C}_{\mathrm{sh}}^{Z}=\hat{C}_{\mathrm{sh}}^{W}=0$. Generalizations that support shear driving at other length scales are straightforward to obtain [e.g., Matthaeus et al., 2004; Breech et al., 2005].

[29] A feature of the present formulation is that the pickup ion effects enter into the $\lambda_{\|}$equation, as is appropriate physically. Equation (13) also involves the quantity $\lambda_{\text {res }}$, which is the (parallel) length scale at which the waves resonate with pickup ions. We are assuming that the energy injected by the pickup scattering process appears mainly in the spectrum of the wave-like component, at the $\lambda_{\|}$corresponding to this resonance. We take $\lambda_{\text {res }}=2 \pi U / \Omega_{p}$, where $\Omega_{p}$ is the proton cyclotron frequency in the Parker spiral magnetic field [Isenberg, 2005; Isenberg et al., 2010b]. At larger distances, $\lambda_{\text {res }}$ is essentially linear in $r$. The final term in equation (13) models the pickup ion energy injection as causing $\lambda_{\|}$to relax toward $\lambda_{\text {res }}$ on the time scale associated with the rate of energy injection. As noted in section 1, this novel aspect of the present model significantly improves the handling of pickup ion physics by allowing pickup ions to directly influence quasi-parallel wave vector fluctuations. This is not the case for some earlier models [e.g., Zank et al., 1996; Matthaeus et al., 1999, 2004; Smith et al., 2001, 2006; Isenberg et al., 2003; Breech et al., 2005, 2008].

[30] Note that the strength of the Alfven speed enters the model equations in several different ways. First, transport terms of order $V_{A} / U$ have been neglected since this is a small parameter beyond about $0.3 \mathrm{AU}$, as discussed by Zank et al. [1996]. Second, in the turbulence modeling terms, the strength of $V_{A}$ is unconstrained compared to the characteristic fluctuation amplitudes $Z$ and $W$. There is no contradiction here since $U \gg Z, W$. Third, because of the scale-separation approach, $V_{A}(r)$ and the mass density vary with the largescale coordinate $r$ but are treated as uniform with respect to the small-scale coordinates (which are averaged over each large-scale distance). Fourth, the pickup ion driving depends on $V_{A}$ [Isenberg et al., 2003; Isenberg, 2005].

[31] The final equation in the model is that for the proton temperature, $T$. It includes adiabatic expansion and heating due to the dissipation of the fluctuations via both $Z$ and $W$ cascades,

$$
\begin{aligned}
\frac{\mathrm{d} T}{\mathrm{~d} r}= & -\frac{4 T}{3 r}+\frac{m_{\mathrm{p}}}{3 U k_{\mathrm{B}}}\left[\alpha\left(\frac{f Z^{3}}{\ell}+\frac{2 f_{z w}^{+}}{1+Z / W} \frac{W Z^{2}}{\ell}\right)\right. \\
& \left.+2 \tilde{\alpha}\left(\frac{\tilde{f}}{1+\lambda / \ell} \frac{Z W^{2}}{\lambda}+\left(1-\tilde{\sigma}_{c}^{2}\right) \frac{W^{4} \lambda_{\|}}{\lambda^{2} V_{A}}\right)\right],
\end{aligned}
$$

where $m_{\mathrm{p}}$ is the proton mass and $k_{\mathrm{B}}$ is the Boltzmann constant. As is typical in solar wind research, we ignore the effects of heat conduction on the proton temperature, and thus its associated transport term is absent from equation (14). Note that the present model and its antecedents are single-fluid proton models and therefore stand in contrast to single-fluid models that in effect add the electron and proton energy equations [e.g., Cranmer and van Ballegooijen, 2005]. Detailed study shows that interchange of energy between electrons and protons is very slow in the solar wind [Breech et al., 2009; Cranmer et al., 2009].

[32] This completes the development of the model, which is seen to consist of equations (7)-(14). Compared to singlecomponent models, there are both more equations and more terms per equation. As far as the contributions of the terms from the nonlinear phenomenology are concerned, some of these will be rather weak for typical solar wind conditions. This includes the IK-type terms (proportional to $1 / V_{A}$ ) and the terms where the $f$ and $f^{\prime}$ functions have a subscript (e.g., $f_{z w}^{+}$).

\section{Numerical Solutions}

[33] To obtain numerical solutions to the model, we make use of a standard numerical package for ordinary differential equations. We compute solutions relevant to (near) ecliptic parameters and compare them with Voyager and Helios observations.

[34] In assigning values to the model's parameters, we are guided by observations. The (uniform) solar wind speed is set at $U=440 \mathrm{~km} \mathrm{~s}^{-1}, V_{A}(1 \mathrm{AU})=51 \mathrm{~km} \mathrm{~s}^{-1}$, and the proton density is assumed to fall off as $1 / r^{2}$. Together these determine $V_{A}(r)$, assuming a Parker spiral field. Observations indicate that the normalized energy difference is approximately constant with radius [Roberts et al., 1987a; Perri and Balogh, 2010]. Furthermore, Milano et al. [2004] reported that at $1 \mathrm{AU}$ the energy difference is nearly isotropic with respect to the magnetic field direction; that is, it does not vary strongly with the field winding angle $\psi$. Hence, we set $\sigma_{D}=\tilde{\sigma}_{D}=-1 / 3$. The value of the shear constants has been extensively discussed by Breech et al. [2008]; we take $C_{\mathrm{sh}}^{Z}=$ $C_{\mathrm{sh}}^{W}=1$. Also, in keeping with recent studies, we adopt de Kármán-Taylor constants $\alpha=2 \beta=0.25$ and $\tilde{\alpha}=2 \tilde{\beta}=0.25$ [Pearson et al., 2004; Breech et al., 2009]. The form used for pickup ion driving,

$$
\frac{\dot{E}_{\mathrm{PI}}}{U}=\zeta \frac{U n_{H}}{\tau_{\text {ion }} n_{\mathrm{SW}}} \exp \left(-L_{\text {cavity }} / r\right)
$$



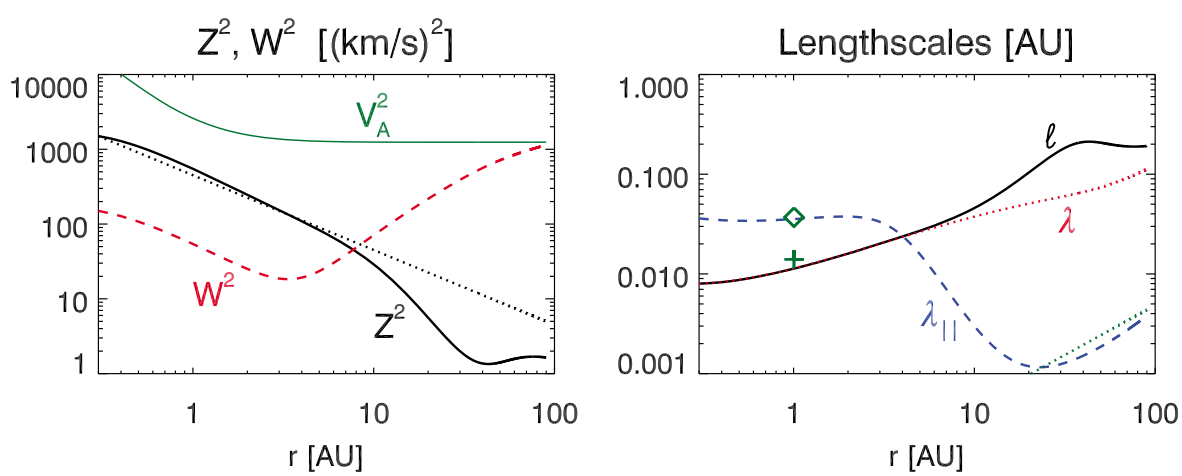

Figure 1. Sample numerical solutions to the transport model with $\zeta=\zeta_{0}=0.04$. The boundary values are $Z^{2}=1500 \mathrm{~km}^{2} \mathrm{~s}^{-2}, W^{2}=150 \mathrm{~km}^{2} \mathrm{~s}^{-2}, \ell=\lambda=0.008 \mathrm{AU}, \lambda_{\|}=0.036 \mathrm{AU}, \sigma_{c}=\tilde{\sigma}_{c}=0.6$, and $T=1.6 \times 10^{6} \mathrm{~K}$. Also shown, for reference, are (left) $V_{A}^{2}$ (dashed curve) and the WKB solution for $Z^{2}$ (dotted curve), and (right) $\lambda_{\text {res }}$ (dotted curve) and observational estimates of the perpendicular and parallel magnetic correlation length at $1 \mathrm{AU}$ (plus sign and diamond [Weygand et al., 2009]).

involves additional parameters, including $L_{\text {cavity }}=5.6 \mathrm{AU}$, $\tau_{\text {ion }}=1.33 \times 10^{6} \mathrm{~s}, n_{H}=0.1 \mathrm{~cm}^{-3}$, and $n_{\mathrm{SW}}(1 \mathrm{AU})=6 \mathrm{~cm}^{-3}$. These parameters are discussed more fully elsewhere [e.g., Isenberg et al., 2003; Isenberg, 2005; Smith et al., 2006]. The quantity $\zeta$ represents the fraction of the ostensibly available pickup ion energy that is actually transferred to the fluctuations, and it varies with $r$. In section 3.1 we ignore this radial dependence and approximate $\zeta$ by the constant value $\zeta_{0}=$ 0.04 . The more accurate approach is used in section 3.2.

\subsection{Solutions With a Single Set of Boundary Conditions}

[35] It remains to choose the boundary conditions for the model. We set the inner boundary at $r=0.3 \mathrm{AU}$, which coincides with the Helios perihelion as well as being far enough out that $V_{A} / U$ is small. The actual boundary values used for $Z^{2}, \ell, \sigma_{c}$, etc., are given in the figure captions. Some general remarks about how they were chosen are appropriate.

[36] Helios data are used as a guide for the range of reasonable values for the total fluctuation energy and cross helicity [e.g., Bavassano et al., 1982; Bruno and Dobrowolny, 1986] from 0.3 to $1 \mathrm{AU}$. Observational studies at $1 \mathrm{AU}$ suggest that the quasi-2-D energy is $\sim 80 \%$ of the total fluctuation energy [Bieber et al., 1996; Horbury et al., 2008; Osman and Horbury, 2009; MacBride et al., 2010], and we use a similar partitioning to set $Z^{2}$ and $W^{2}$ here.

[37] Unfortunately, no observational values for $\ell$ and $\lambda$ are available, since the two-component decomposition is difficult to perform with current data sets. As guidelines for their boundary values, we use observed magnetic correlation lengths. For example, Bruno and Dobrowolny [1986] obtained $\ell_{\mathrm{cor}}^{b} \approx 0.0067 \mathrm{AU}$ at $0.3 \mathrm{AU}$ and $\ell_{\mathrm{cor}}^{b} \approx 0.013 \mathrm{AU}$ at $1 \mathrm{AU}$, although these values are from the trailing edges of high-speed streams and thus not necessarily representative of typical ecliptic conditions. Here $\ell_{\mathrm{cor}}^{b}$ is the integral of the magnetic correlation function divided by its value at zero lag. At $1 \mathrm{AU}$, the usual situation appears to be that the velocity correlation length is longer than the magnetic one [Matthaeus and Goldstein, 1982; Wicks et al., 2010]. The boundary value of $\lambda_{\|}$is not crucial, and large variations in it produce very little change in the solutions.
[38] There is also an abundance of data at $1 \mathrm{AU}$ for fluctuation energy, cross helicity, and correlation length. We thus attempt to adjust the boundary conditions so that the solutions are also consistent with $1 \mathrm{AU}$ data. For example, Weygand et al. [2009] used Cluster data sets to estimate perpendicular and parallel magnetic correlation lengths in slow wind, obtaining 0.014 AU and 0.037 AU, respectively.

[39] In Figure 1 we display numerical solutions for a representative set of boundary values. Initially, both energies decrease and their perpendicular length scales increase, with $\lambda_{\|}$approximately constant. The shear driving causes $Z^{2}$ to be above the level of the corresponding WKB solution (dotted curve), although the behavior is still WKB-like within $\sim 5$ AU. The situation for $W^{2}$ is similar. Beyond $5 \mathrm{AU}$, shear driving is weak and the pickup ion driving is important, and the $Z^{2}$ and $W^{2}$ profiles depart strongly from WKB behavior.

[40] Perhaps the most striking feature of Figure 1 (left) is the large increase in wave-like energy which occurs for $r \gtrsim$ $4 \mathrm{AU}$ and is due to the pickup ion driving. Indeed, for $r \gtrsim$ 9 AU, the wave-like energy is dominant, and it would be very interesting to perform Bieber-type analyses [Bieber et al., 1996] at these larger distances, in order to see if such a reversal of the quasi-2-D to wave-like preponderance is observed. Smith [2003] has performed such a study using Ulysses data at various distances and heliolatitudes. Restricting attention to the low-latitude results from Smith [2003] and from the study by Bieber et al. [1996], one sees that these results can be interpreted as indicating that larger distances are associated with increasing energy in the slab component. For example, the slab fraction was often $\gtrsim 50 \%$ in 1992 when Ulysses was at $\sim 5$ AU and low latitudes. This is similar to the behavior shown in Figure 1. (For discussion of the influence of latitude on transport of solar wind fluctuations, see Smith [2003] and Breech et al. [2008].)

[41] Figure 1 also shows the length scale evolutions, with $\ell$ and $\lambda$ both increasing rather smoothly out to $r \approx 30 \mathrm{AU}$, after which $\ell$ decreases somewhat due to the increase of $Z^{2}$ via transfer (exchange) from $W^{2}$. Convergence of $\lambda_{\|}$toward $\lambda_{\text {res }}$ is also evident at distances where pickup ion driving is important.

[42] It is, of course, also important to compare quantities derived from the numerical solutions to observational values. 

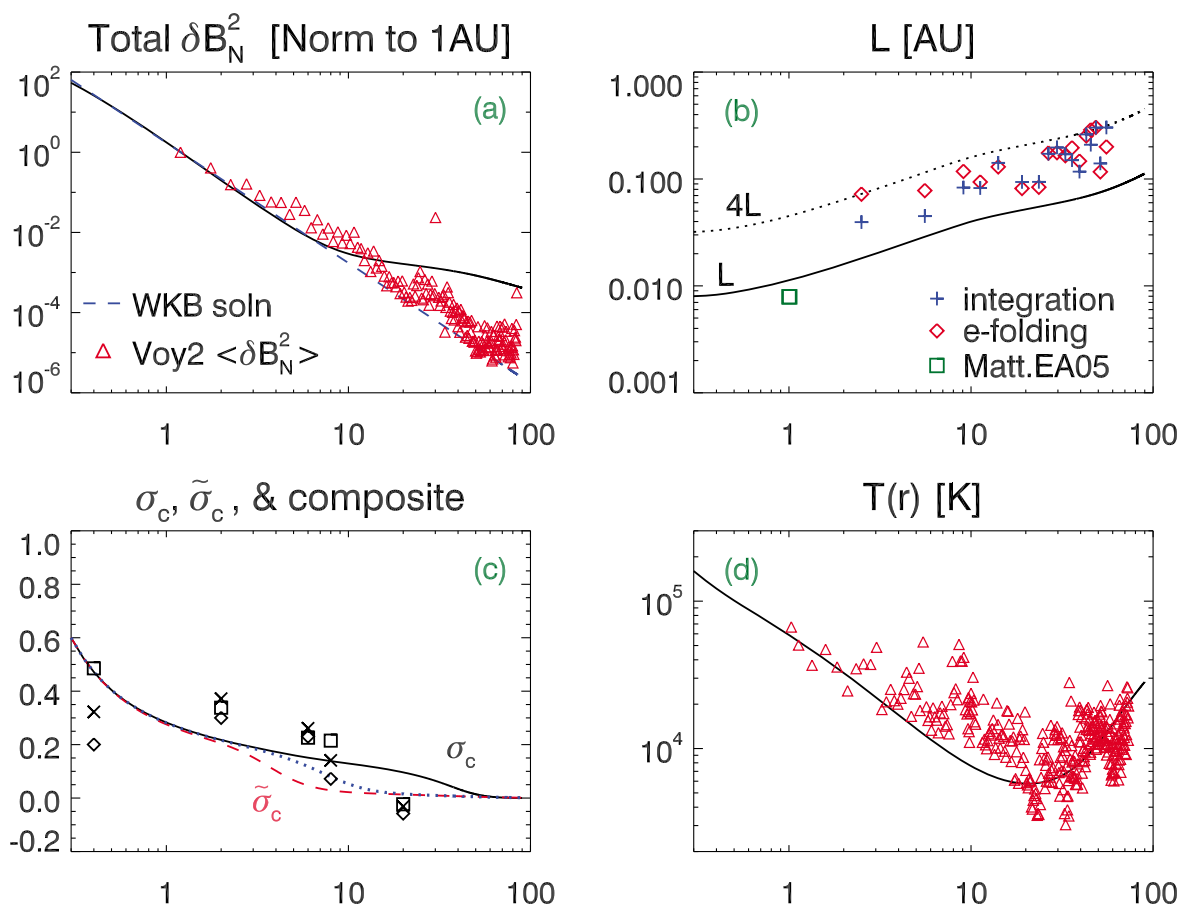

Figure 2. Comparison of the sample numerical solution with observational data. The horizontal axis is heliocentric distance in astronomical units. See Smith et al. [2006] for details regarding calculation of the Voyager 2 observational values. (a) The Voyager magnetic field values are for the $\mathrm{N}$ component of the field, in RTN coordinates, and all values are normalized to the level at 1 AU. See equation (16). (b) $L=\left(Z^{2} \ell+\right.$ $\left.W^{2} \lambda\right) /\left(Z^{2}+W^{2}\right)$ is the composite energy-weighted length scale; see equation (17). (c) The composite cross helicity is the dotted curve, and observational values are 3, 9, and $27 \mathrm{~h}$ averages, courtesy of D. A. Roberts [Roberts et al., 1987b]. Temperature data are courtesy of J. D. Richardson.

In Figure $2 \mathrm{a}$ the variance of the $N$ component (RTN coordinates) of the fluctuating magnetic field from Voyager 2 data is plotted, as triangles. The solid curve is the model-based estimate for this quantity,

$$
\delta B_{N}^{2}=\frac{1}{2} 4 \pi \rho\left(Z^{2}+W^{2}\right) \frac{1-\sigma_{D}}{2}=\frac{4 \pi \rho}{3}\left(Z^{2}+W^{2}\right)
$$

where we assume that the $N$ component is half the total magnetic variance [Smith et al., 2006; Isenberg et al., 2010a]; the proton mass density is $\rho=n_{\mathrm{SW}} m_{\mathrm{p}}$. For plotting purposes, both the observational values and the model $\delta B_{N}^{2}$ are normalized by their $1 \mathrm{AU}$ values. While the agreement is encouraging for $r \lesssim 10 \mathrm{AU}$, it worsens thereafter, with the model solution significantly above the observed level. Intriguingly, the WKB solution (dashed curve) provides a useful lower limit to the data, despite the manifestly nonWKB behavior of $Z^{2}$ and $W^{2}$ beyond $\sim 10$ AU (Figure 1).

[43] There are several issues associated with comparing observational values of the correlation length to the numerical solutions for $\ell$ and $\lambda$. Voyager data can be used to calculate estimates of the magnetic correlation length of the magnetic field fluctuations. This usually yields the radial correlation length (i.e., in the solar wind flow direction). Beyond $\approx 10 \mathrm{AU}$ this corresponds to a perpendicular correlation length, which is suitable for comparison with solutions to the present model. Inside $10 \mathrm{AU}$, however, the observational correlation lengths are typically neither perpendicular ones nor parallel ones, so the data/model comparison needs to be considered carefully. Moreover, the correlation scale is a sensitive and sometimes difficult determination. (See Smith et al. [2001, 2006] and Isenberg et al. [2010a] for details on how the observational values are calculated.)

[44] Unfortunately, separate determinations of $\ell$ and $\lambda$ are usually not available. Hence, we use the model solutions to construct a composite "center of mass"-style length:

$$
L=\frac{Z^{2} \ell+W^{2} \lambda}{Z^{2}+W^{2}}
$$

This is equivalent to the perpendicular correlation length for the total fluctuation. As Figure $2 \mathrm{~b}$ indicates, the agreement between $L$ and the Voyager magnetic correlation lengths (averaged into 3 AU bins) is good and is a significant improvement to the results from single-component models [e.g., Matthaeus et al., 1999; Smith et al., 2001; Isenberg et al., 2003]. Also shown in Figure 2b is the curve $4 L$, which is seen to be a rough upper limit to the observational values. This can be understood by recalling the recent result [Matthaeus et al., 2005] that at 1 AU single-spacecraft measurements appear to overestimate the true correlation length by factors of 2-4, where "true" means as determined using data sets from multiple spacecraft.

[45] In Figure 2c, we display observational values of the normalized cross helicity [Roberts et al., 1987b] along with the model solutions for $\sigma_{c}, \tilde{\sigma}_{c}$, and their energy-weighted 


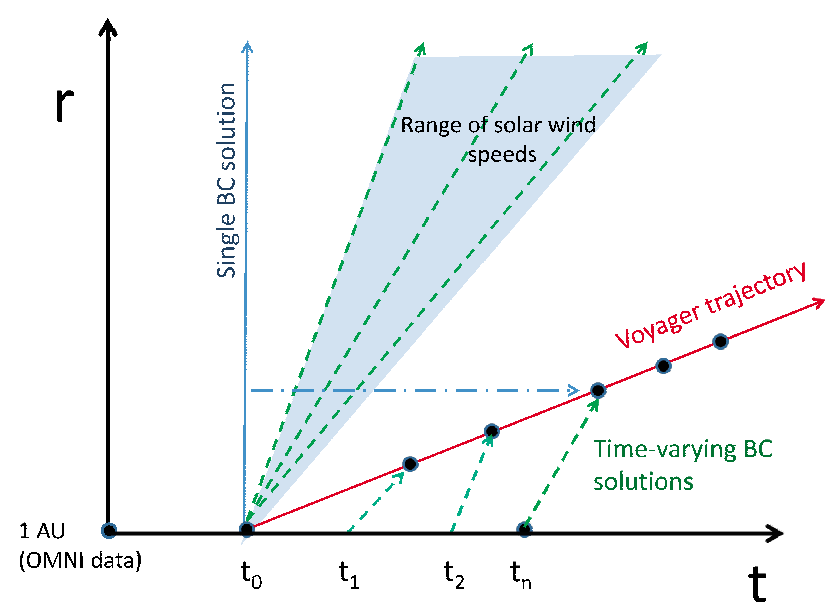

Figure 3. Diagram showing the relationship between Voyager measurements and the two types of solution computed herein, namely, a solution with a single set of boundary conditions and a "piecewise" solution computed using boundary conditions obtained from 1 AU OMNIWeb averages at times appropriately lagged with respect to the Voyager measurements at larger distances. Dashed (green) lines represent solar wind speeds.

combined version $\left(Z^{2} \sigma_{c}+W^{2} \tilde{\sigma}_{c}\right) /\left(Z^{2}+W^{2}\right)$. The combined version (Figure 2c, dotted curve) tends to undershoot the observations. Both $\sigma_{c}$ and $\tilde{\sigma}_{c}$ decrease smoothly toward zero with distance. For $r \lesssim 2 \mathrm{AU}$ the decrease is largely due to shear driving, which injects equal amounts of energy into the \pm fluctuations and is strong enough to overcome the dynamic alignment terms in equations (9) and (10) [Matthaeus et al., 2004]. At larger $r$, pickup ion driving - which is present only in the $\tilde{\sigma}_{c}$ equation-pulls $\tilde{\sigma}_{c}$ to zero many astronomical units before $\sigma_{c}$ gets there.

[46] As far as the proton temperature is concerned, the numerical solution reproduces the qualitative behavior of the observations, including the increasing temperature beyond $\sim 30$ AU. From 1 to $2 \mathrm{AU}$, the model solution mirrors the observational $T(r)$ in an encouraging way.

[47] We now briefly consider the sensitivity of the model to changes in the shear driving. As is to be expected, increasing the shear strength leads to a less rapid falloff of $Z^{2}(r)$. For $C_{\mathrm{sh}}^{Z} \approx 1.1-2$, this tends to improve the agreement between the magnetic energy profile and the Voyager 2 observations out to $\sim 10 \mathrm{AU}$ (see Figure 2a). These values of $C_{\mathrm{sh}}^{Z}$ also produce a proton temperature profile that is in better accord with observations, provided the boundary value for $T$ is reduced. On the other hand, stronger shear causes the model profile for $\sigma_{c}$ to tend to zero within a few astronomical units, which somewhat worsens agreement with the (limited number of) observational data points.

[48] We remind readers that there are complications associated with evaluating the quality of solutions in the outer heliosphere. For example, beyond $\sim 30 \mathrm{AU}$, Voyager is not in the ecliptic, whereas the parameters for our model are chosen to be appropriate for (near) the ecliptic. In particular, for $r \approx 40-55 \mathrm{AU}$, Voyager 2 experienced conditions associated with solar minimum that are not well represented using parameters measured near Earth [Smith et al., 2006]. More- over, during the transit of Voyager over tens of astronomical units, the inner heliospheric conditions are strongly variable, but the numerical solutions use steady boundary conditions. This casts doubt on the fidelity of a transport solution based on a single set of boundary data, since the boundary data themselves have a broad distribution over the time scales of interest [e.g., Smith et al., 2001].

[49] We conclude that the comparison of model results and observations in the present section is acceptable. However, we are motivated to improve two significant deficiencies of the model: (1) the use of a single set of boundary conditions, which cannot account for the varying solar wind plasma conditions at Voyager 2, and (2) the simplified approach to the pickup ion driving, in which $\zeta=\zeta_{0}$ is taken to be constant [Smith et al., 2006]. Both these limitations are addressed in section 3.2 .

\subsection{Solutions With Time-Varying Boundary Conditions}

[50] The shortcomings of models that employ fixed inner heliospheric boundary conditions have been addressed previously in the context of single-component models [Richardson and Smith, 2003; Smith et al., 2006; Isenberg et al., 2010a]. Instead of using a single set of boundary data to generate a solution to be compared with the observations along the Voyager trajectory, these authors compute a separate solution for each (rotation-averaged) Voyager observation. For each of these solutions, 1 AU data, recorded at an earlier time, are used to specify some of the conditions at $1 \mathrm{AU}$, such as fluctuation energy density. By time-lagging the $1 \mathrm{AU}$ observation by the approximate wind transit time to the position of the Voyager observation, an improved solution at that point is obtained. Figure 3 illustrates in diagrammatic form the relationship between the single boundary condition solutions and the time-lagged, variable boundary data solutions. Here we report on solutions to the present two-component model using the time-lagged, variable boundary data approach.

[51] Specifically, here the boundary conditions at $1 \mathrm{AU}$ are chosen to matchup with the advection time to Voyager 2. The Voyager time series is partitioned into nonoverlapping time intervals, each approximately three solar rotation periods in duration. Average values for $\delta B_{N}^{2}, \ell_{\text {cor }}^{b}, T$, and $U$ are computed for each of these intervals, along with the heliocentric distances delimiting the intervals. Then, using the average solar wind speed in each interval, its start and end times are mapped back to $1 \mathrm{AU}$ to give the end points of a corresponding interval there. OMNIWeb data $[$ King and Papitashvili, 1994] are used to compute average values for $Z^{2}+W^{2}, T, V_{A}$, and the solar wind density and speed over this 1 AU interval. These values, together with fixed estimates for the normalized cross helicities and length scales at $1 \mathrm{AU}$, provide a full set of boundary conditions. The model is solved for each set of boundary conditions, and the solution is saved only for the distance range corresponding to the Voyager interval it was mapped back from. This gives a "piecewise" solution that can be compared to the (three solar-rotationaveraged) observations (see Figure 3).

[52] Unfortunately, OMNIWeb data are not usually suitable for estimating correlation lengths [Smith et al., 2006]. Instead, estimates for the length scales at $1 \mathrm{AU}$ are taken from 

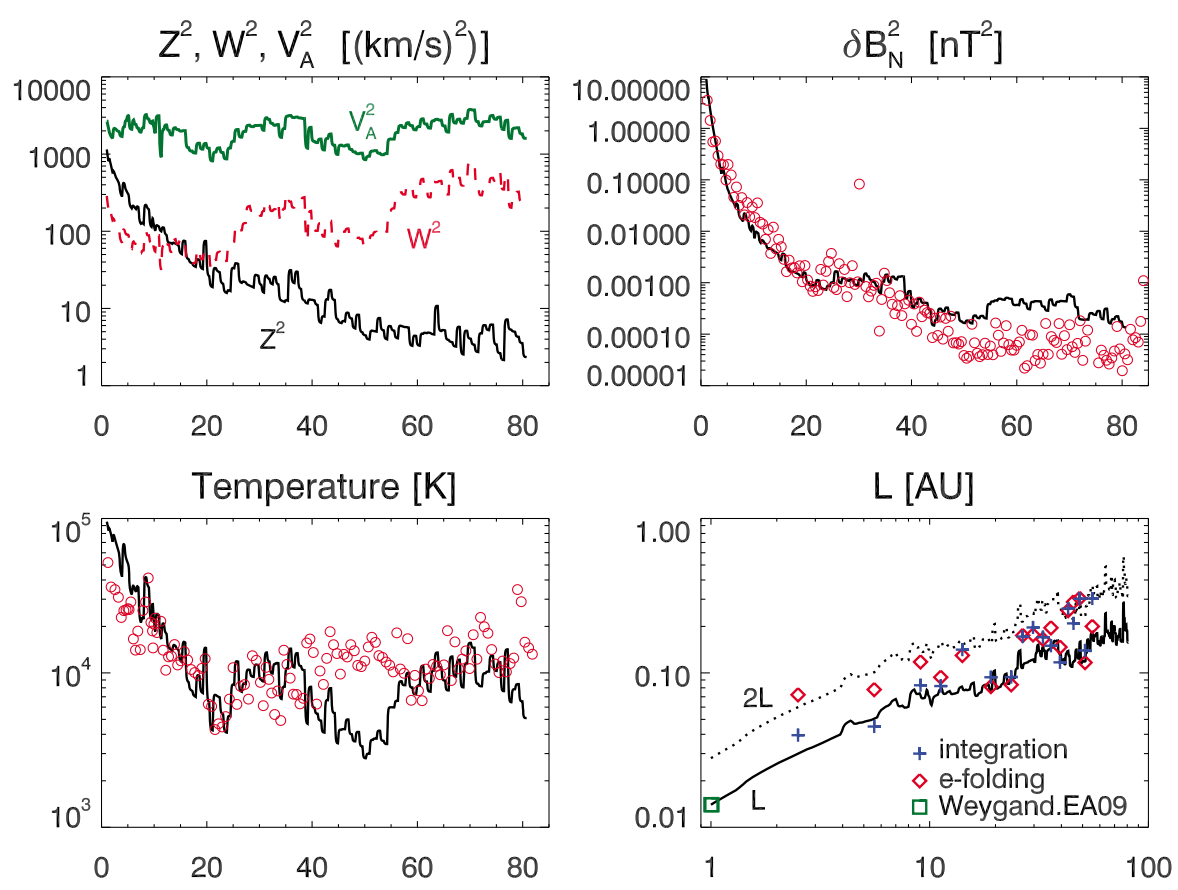

Figure 4. Comparison of the "piecewise" numerical solution (curves) discussed in section 3.2 with observational data from Voyager 2, with averages over three solar rotations indicated by open circles. The horizontal axis is heliocentric distance in astronomical units. The solutions to the model have been smoothed using a five-point boxcar average, and length scale data values have been collected into 3 AU bins.

an analysis of Cluster data: $\ell=\lambda=0.014 \mathrm{AU}, \lambda_{\|}=0.037 \mathrm{AU}$ [Weygand et al., 2009]. We choose $\sigma_{c}=\tilde{\sigma}_{c}=0.5$, although the model is rather insensitive to the choice since the cross helicities both approach zero by about $10 \mathrm{AU}$ (see Figure 2c). Note that the partitioning of the total fluctuation energy into its $Z^{2}$ and $W^{2}$ components must also be specified. Here we assume that $80 \%$ of the fluctuation energy is in the quasi-2-D component [Bieber et al., 1996; Osman and Horbury, 2009]. This percentage of 2-D fluctuations is most appropriate to slow wind [Dasso et al., 2005].

[53] In conjunction with the varying boundary conditions, we also use a more accurate form of the pickup ion driving, developed by Isenberg et al. [2003] and Isenberg [2005]. The fraction of the pickup ion energy that goes into the turbulence $(\zeta)$ depends on $V_{A} / U, W / V_{A}$, and the energy spectrum of the wave-like component, which is assumed here to have an inertial range with a $-5 / 3$ power law.

[54] As Figure 4 shows, this approach produces improved agreement between the model solutions and the observational values. The modulation of the pickup ion driving of $W^{2}$ by the Alfvén speed is evident in the top left panel [Smith et al., 2006]. For the proton temperature, the most obvious region of disagreement is the 40-55 AU range. During that period, Voyager 2 was at high latitude and experiencing solar minimum conditions, which are unlikely to be predicted well using solar wind data from $1 \mathrm{AU}$ as boundary conditions [Smith et al., 2006]. Interestingly, for the present model $\delta B_{N}^{2}$ observations are reasonably well predicted for $r \lesssim 50 \mathrm{AU}$, although the prediction is too high beyond that. Perhaps the observational quantity predicted best by the model solutions is the magnetic correlation length, for which the agreement is better by a factor of 2 almost everywhere. For each quantity, there are regions where even some relatively fine-scale var- iations in the observations are accounted for in the model solutions.

\section{Summary and Conclusions}

[55] A transport model for solar wind fluctuations has been formulated, in which the fluctuations are treated as two coupled, incompressible components, specifically a quasi2-D piece and a wave-like piece. In addition to the usual expansion, mixing, and nonlinear effects, the present model includes exchange terms between the fluctuation components and the placement of the driving term due to pickup ion scattering in the wave-like fluctuation equations. The latter improvement is in accord with theories of wave-particle interaction that suggest that the principal pickup driving is at high-parallel wavenumber [e.g., Isenberg et al., 2003]. Recent observations by Joyce et al. [2010] also support this conclusion.

[56] The example solutions show that the two-component transport equations can provide reasonable solutions for temperature, cross helicity, and (composite) correlation scale. The fluctuation amplitude behaves well out to $\sim 30 \mathrm{AU}$, with pickup driving very important beyond $\sim 10 \mathrm{AU}$. At larger distances the observations are usually significantly below the level predicted by the theoretical model. However, we note that the strong wave-driving in the outer heliosphere will excite wave vectors mainly in the azimuthal direction, perpendicular to the radial frozen-in flow observations at the spacecraft. It is therefore possible that at least part of this discrepancy is due to lack of observations of the relevant quantities.

[57] Observational results [Milano et al., 2004] suggest that there is strong "connectedness" or coupling of presumptive 
$Z$ and $W$ components of the turbulence, at least as far as observed normalized quantities like $\sigma_{c}$ and $\sigma_{D}$ are concerned. On the other hand, the solutions presented here show that there is still plenty of scope for the energy and normalized cross helicity of each component to have quite different values at some heliocentric distances. More, and higherquality, observations would assuredly improve understanding of these differences and similarities. Finally, we note that extensions to the model are being pursued, including modifications to support variable solar wind speed [Smith et al., 2006; Isenberg et al., 2010a] and to account for separate length scales for each Elsässer field.

\section{Appendix A: Two-Component Phenomenology for Incompressible Homogeneous MHD Turbulence With Cross Helicity}

[58] For completeness, we state below the equations for our phenomenology for the evolution of incompressible homogeneous two-component turbulence with cross helicity (i.e., $H_{c} \neq 0$ ). These equations have not been reported elsewhere in the literature. Although they are a straightforward generalization of the $H_{c}=0$ case derived by Oughton et al. [2006], they appear rather more cumbersome, since there are twice as many equations. As noted in section 2.1, however, the structure of the terms is unchanged from the simpler $H_{c}=0$ case, except for the appearance of crosshelicity-dependent attenuation factors on many terms (the $f$ functions).

[59] The first step is to generalize equations (17) and (18) of Oughton et al. [2006] to the $H_{c} \neq 0$ situation:

$$
\begin{gathered}
\frac{1}{2} \frac{\mathrm{d} Z_{ \pm}^{2}}{\mathrm{~d} t}=-Z_{ \pm}^{2} \frac{Z_{\mp}}{2 \ell}-Z_{ \pm}^{2} \frac{W_{\mp}}{\ell} \Gamma_{z}^{w_{\mp} z_{ \pm}}+Y^{ \pm} \\
\frac{1}{2} \frac{\mathrm{d} W_{ \pm}^{2}}{\mathrm{~d} t}=-W_{ \pm}^{2} \frac{Z_{\mp}}{\lambda} \Gamma_{z}^{z_{\mp} w_{ \pm}}-W_{ \pm}^{2} \frac{W_{\mp}}{\lambda} \Gamma_{w}^{w_{\mp} w_{ \pm}}-Y^{ \pm}, \\
Y^{ \pm}=W_{ \pm} Z_{ \pm}\left[\frac{Z_{\mp}}{\lambda} \Gamma_{w}^{z_{\mp} w_{ \pm}}+\frac{W_{\mp}}{\lambda} \Gamma_{w}^{w_{\mp} w_{ \pm}}-\frac{Z_{\mp}}{\ell} \Gamma_{w}^{z_{\mp} z_{ \pm}}-\frac{W_{\mp}}{\ell} \Gamma_{w}^{w_{\mp} z_{ \pm}}\right],
\end{gathered}
$$

where the assumptions $\ell_{+} \approx \ell_{-} \approx \ell$ and $\lambda_{+} \approx \lambda_{-} \approx \lambda$ are made. The $Y^{ \pm}$terms model exchange of excitation between the $Z$ and $W$ components. The factors

$$
\Gamma_{c}^{a b}=\frac{1}{1+\tau_{\mathrm{nl}}^{a b} / \tau_{A}^{c}}
$$

are associated with the decay rate of the triple correlation for the term being modeled and involve the nonlinear $\left(\tau_{\mathrm{nl}}\right)$ and Alfvén $\left(\tau_{A}\right)$ time scales. The superscripts $a, b, c$ represent either the $Z$ or the $W$ component. For example, $\tau_{\mathrm{nl}}^{Z W}=\lambda / Z$, $\tau_{\mathrm{nl}}^{W Z}=\ell / W$, and $\tau_{A}^{W}=\lambda_{\|} / V_{A}$. (See Oughton et al. [2006] for further details.)

[60] In fact, these equations are the desired phenomenology. For our purposes it is convenient to express them in terms of the energy and cross helicity of each component. Define $2 Z^{2}=Z_{+}^{2}+Z_{-}^{2}$ and $4 H_{c}^{Z}=Z_{+}^{2}-Z_{-}^{2}$, with analogous forms for the $W$ component (see Table 1). The kinetic plus magnetic energy (per volume) of the fluctuations is then $E^{v}+E^{b}=\left(Z^{2}+W^{2}\right) / 2$. The normalized cross helicities are $\sigma_{c}=\left(Z_{+}^{2}-Z_{-}^{2}\right) /\left(Z_{+}^{2}+Z_{-}^{2}\right) \equiv 2 H_{c}^{Z} / Z^{2}$ and $\tilde{\sigma}_{c}=2 H_{c}^{W} / W^{2}$.

[61] Adding and subtracting the $Z_{+}^{2}$ and $Z_{-}^{2}$ equations gives equations for the $Z$ component's total energy and cross helicity, and the same is true for the $W$ component. Using the identities $Z_{ \pm}^{2}=\left(1 \pm \sigma_{c}\right) Z^{2}$ and $W_{ \pm}^{2}=\left(1 \pm \tilde{\sigma}_{c}\right) W^{2}$, we obtain

$$
\begin{gathered}
\frac{1}{2} \frac{\mathrm{d} Z^{2}}{\mathrm{~d} t}=-\alpha\left[\frac{Z^{3}}{2 \ell} f_{z z}^{+}+\frac{W Z^{2}}{\ell} \frac{f_{z w}^{+}}{1+Z / W}-X^{+}\right] \\
\frac{\mathrm{d} H_{c}^{Z}}{\mathrm{~d} t}=-\alpha\left[\frac{Z^{3}}{\ell \ell} f_{z z}^{-}+\frac{W Z^{2}}{\ell} \frac{f_{z w}^{-}}{1+Z / W}-X^{-}\right] \\
\frac{1}{2} \frac{\mathrm{d} W^{2}}{\mathrm{~d} t}=-\tilde{\alpha}\left[\frac{W^{2} Z}{\lambda} \frac{f_{w z}^{+}}{1+\lambda / \ell}+\frac{W^{4} \lambda_{\|}}{\lambda^{2} V_{A}}\left(1-\tilde{\sigma}_{c}^{2}\right)\right]-\alpha X^{+}, \\
\frac{\mathrm{d} H_{c}^{W}}{\mathrm{~d} t}=-\tilde{\alpha}\left[\frac{W^{2} Z}{\lambda} \frac{f_{w z}^{-}}{1+\lambda / \ell}\right]-\alpha X^{-}, \\
X^{ \pm}=\left(Y^{+} \pm Y^{-}\right) / 2 \approx \frac{\lambda_{\|}}{V_{A}}\left(\frac{1}{\lambda^{2}}-\frac{1}{\ell^{2}}\right) W Z\left[Z^{2} f_{X, z}^{ \pm} \pm W^{2} f_{X, w}^{ \pm}\right] \\
2 f_{a b}^{ \pm}=\left(1+\sigma^{a}\right) \sqrt{1-\sigma^{b}} \pm\left(1-\sigma^{a}\right) \sqrt{1+\sigma^{b}} \\
2 f_{X, a}^{ \pm}=\sqrt{1-\left(\sigma^{a}\right)^{2}}\left[\sqrt{1+\tilde{\sigma}_{c}} \sqrt{1-\sigma_{c}} \pm \sqrt{1-\tilde{\sigma}_{c}} \sqrt{1+\sigma_{c}}\right]
\end{gathered}
$$

with $\sigma^{a} \equiv \sigma_{c}$ or $\tilde{\sigma}_{c}$ for the component $a=Z$ or $W$. The $\Gamma_{c}^{a b}$ factors that have a subscript $w$ have been approximated as $\tau_{A}^{c} / \tau_{\mathrm{nl}}^{a b} \ll 1$. Here we have introduced several $f$ functions, which depend on the normalized cross helicities. Since these $f$ functions decrease toward zero as the cross helicities increase in magnitude, they are, in effect, attentuation factors on the (modeled) nonlinear terms. Moreover, as the cross helicities approach zero, all the $f^{+} \rightarrow 1$ and all the $f^{-} \rightarrow 0$, leading to recovery of the zero-cross-helicity form for the twocomponent phenomenology, given above as equations (4) and (5). In the main body of the paper we use the simplified notation $f=f_{z z}^{+}$and $\tilde{f}=f_{w z}^{+}$.

[62] Since equations (A5)-(A8) are a phenomenological model, each term on the RHS can be argued to have an $O(1)$ constant multiplying it, included here as the factors $\alpha$ and $\tilde{\alpha}$. These constants are not completely free since, for example, when adding the $Z^{2}$ and $W^{2}$ equations, we should require the exchange terms to exactly cancel. Even with such restrictions, many terms could in principle have distinct multiplying constants. Here, however, we have adopted the simpler approach of using the same constant for all terms in a given equation (except for the variations required in connection with the exchange terms).

[63] Equations for the normalized cross helicities (not shown) are obtained by differentiating their definitions, $\sigma_{c}=$ $2 H_{c}^{Z} / Z^{2}$ and $\tilde{\sigma}_{c}=2 H_{c}^{W} / W^{2}$, and then using equations (A5)(A8). It is then convenient to define various $f^{\prime}$ functions, 
such as $f^{\prime} \equiv f_{z z}^{\prime}=\sigma_{c} f_{z z}^{+}-f_{z z}^{-}, \tilde{f}^{\prime} \equiv f_{w z}^{\prime}=\tilde{\sigma}_{c} f_{w z}^{+}-f_{w z}^{-}$, and $f_{z w}^{\prime}=\sigma_{c} f_{z w}^{+}-f_{z w}^{-}$, akin to those introduced for singlecomponent models by Matthaeus et al. [2004].

[64] The length scale evolution equations are derived just as they were by Oughton et al. [2006]. Those for $\ell$ and $\lambda$ follow from the conservation laws $\ell Z^{2 \beta / \alpha}=$ const and $\lambda W^{2 \tilde{\beta} / \tilde{\alpha}}=$ const. These are analogous to the conservation laws that hold for the de Kármán-Howarth phenomenology for NavierStokes turbulence. One sees that the values $2 \beta / \alpha=1$ and 2 (like those for $2 \tilde{\beta} / \tilde{\alpha}$ ) are of particular physical relevance [Zank et al., 1996; Matthaeus et al., 1996]. The former is associated with decay at constant Reynolds numbers, and the latter is associated with decay subject to constant area under the sum of the $\boldsymbol{v}$ and $\boldsymbol{b}$ correlation functions. Differentiating the conservation laws produces

$$
\begin{gathered}
\frac{\mathrm{d} \ell}{\mathrm{d} t}=-\frac{\beta}{\alpha} \frac{\ell}{Z^{2}} \frac{\mathrm{d} Z^{2}}{\mathrm{~d} t}, \\
\frac{\mathrm{d} \lambda}{\mathrm{d} t}=-\frac{\tilde{\beta}}{\tilde{\alpha}} \frac{\lambda}{W^{2}} \frac{\mathrm{d} W^{2}}{\mathrm{~d} t} .
\end{gathered}
$$

Operationally, $\beta$ and $\tilde{\beta}$ can also be interpreted as the $O(1)$ constants multiplying the RHS of the length scale equations, as becomes apparent after substitution of equation (A5) in equation (A11), etc. Note that the conservation laws hold for the homogeneous two-component phenomenology presented in this appendix. They are not expected to hold in the transport model presented in the main body of the present paper.

[65] The $\lambda_{\|}$evolution, also modeled after Oughton et al. [2006], assumes growth on the time scale associated with the nonresonant interactions (the Iroshnikov-Kraichnan-type term), since the resonant terms do not change the parallel length scale [Shebalin et al., 1983; Oughton et al., 2006]. This yields

$$
\frac{\mathrm{d} \lambda_{\|}}{\mathrm{d} t}=2 \tilde{\alpha} \frac{\lambda_{\|}^{2}}{V_{A}} \frac{W^{2}}{\lambda^{2}}\left(1-\tilde{\sigma}_{c}^{2}\right) .
$$

[66] Finally, we outline a proof of the orthogonality conditions referred to in section 2.2. For definiteness, consider $\left\langle q_{i}^{+} w_{j}^{+}\right\rangle=0$, where angle brackets denote an average over the (small-scale) spatial coordinates $\boldsymbol{x}$. Expressing the fluctuation fields as Fourier expansions

$$
q_{i}^{+}(\boldsymbol{x})=\sum_{\boldsymbol{k} \in \mathbb{Q}} a_{\boldsymbol{k}}(t) e^{i \boldsymbol{k} \cdot \boldsymbol{x}} \quad \text { and } \quad w_{j}^{+}(\boldsymbol{x})=\sum_{\boldsymbol{p} \in \mathbb{W}} b_{\boldsymbol{p}}(t) e^{i \boldsymbol{p} \cdot \boldsymbol{x}}
$$

shows that $\left\langle q_{i}^{+} w_{j}^{+}\right\rangle$depends on $\int e^{i(\boldsymbol{k}+\boldsymbol{p}) \cdot \boldsymbol{x}} \mathrm{d}^{3} x \propto \delta(\boldsymbol{k}+\boldsymbol{p})$. The key point is whether the set of wave vectors for the first field $(\mathbb{Q})$ has any intersection with that for the second field (W). If it does not, then $\delta(\boldsymbol{k}+\boldsymbol{p})=0$. Because the twocomponent decomposition is defined in terms of the time scales associated with the Fourier wave vectors of the fluctuations, $\mathbb{Q}$ and $\mathbb{W}$ are necessarily disjoint sets if the $z$ and $w$ fluctuations are both of the plus type or both of the minus type. It may or may not be the case when $z$ and $w$ are of different types.

[67] This is a more general condition than the one stated by Oughton et al. [2006], who used only traced versions, such as $\left\langle\boldsymbol{q}^{+} \cdot \boldsymbol{w}^{+}\right\rangle$. One can extend the proof to show that if the two fields are orthogonal at zero separation, then they are also orthogonal at finite separation, e.g., $\left\langle q_{i}^{+}(\boldsymbol{x}) w_{j}^{+}(\boldsymbol{x}+\boldsymbol{y})\right\rangle=0$, where $\boldsymbol{y}$ is a fixed spatial offset vector.

[68] Acknowledgments. This research was supported by the University of Waikato Strategic Research Investment Fund, by NASA through the Heliospheric Theory (NNX08AI47G) and SR\&T (NNX07AH75G NNX09AVA49G) programs, by the NSF (SHINE ATM-0752135, ATM0635863), and by the DoE (DEFG0207ER46372). B.A.B.'s research was supported in part by an appointment to the NASA Postdoctoral Program at Goddard Space Flight Center, administered by Oak Ridge Associated Universities through a contract with NASA. Our thanks to John Richardson and the MIT Space Plasma Group for making the Voyager temperature data available online at http://web.mit.edu/space/www/voyager/voyager.html.

[69] Philippa Browning thanks the reviewers for their assistance in evaluating this paper.

\section{References}

Bavassano, B., M. Dobrowolny, F. Mariani, and N. F. Ness (1982) Radial evolution of power spectra of interplanetary Alfvénic turbulence, J. Geophys. Res., 87(A5), 3617-3622, doi:10.1029/JA087iA05p03617.

Belcher, J. W. (1971), Alfvénic wave pressures and the solar wind, Astrophys. J., 168, 509-524.

Belcher, J. W., and L. Davis Jr. (1971), Large-amplitude Alfvén waves in the interplanetary medium: 2, J. Geophys. Res., 76(16), 3534-3563, doi:10.1029/JA076i016p03534.

Bieber, J. W., W. H. Matthaeus, C. W. Smith, W. Wanner, M.-B. Kallenrode, and G. Wibberenz (1994), Proton and electron mean free paths: The Palmer consensus revisited, Astrophys. J., 420, 294-306.

Bieber, J. W., W. Wanner, and W. H. Matthaeus (1996), Dominant twodimensional solar wind turbulence with implications for cosmic ray transport, J. Geophys. Res., 101(A2), 2511-2522, doi:10.1029/95JA02588.

Bondeson, A. (1985), Cascade properties of shear Alfvén turbulence, Phys. Fluids, 28, 2406-2411, doi:10.1063/1.865246.

Breech, B., W. H. Matthaeus, J. Minnie, S. Oughton, S. Parhi, J. W. Bieber, and B. Bavassano (2005), Radial evolution of cross helicity in highlatitude solar wind, Geophys. Res. Lett., 32, L06103, doi:10.1029/ 2004GL022321.

Breech, B., W. H. Matthaeus, J. Minnie, J. W. Bieber, S. Oughton, C. W. Smith, and P. A. Isenberg (2008), Turbulence transport throughout the heliosphere, J. Geophys. Res., 113, A08105, doi:10.1029/2007JA012711.

Breech, B., W. H. Matthaeus, S. R. Cranmer, J. C. Kasper, and S. Oughton (2009), Electron and proton heating by solar wind turbulence, J. Geophys. Res., 114, A09103, doi:10.1029/2009JA014354.

Bruno, R., and M. Dobrowolny (1986), Spectral measurements of magnetic energy and magnetic helicity between 0.29 and 0.97 AU, Ann. Geophys., 4, 17-22.

Coleman, P. J., Jr. (1968), Turbulence, viscosity, and dissipation in the solar wind plasma, Astrophys. J., 153, 371-388.

Cranmer, S. R., and A. A. van Ballegooijen (2003), Alfvénic turbulence in the extended solar corona: Kinetic effects and proton heating, Astrophys. J., 594, 573-591.

Cranmer, S. R., and A. A. van Ballegooijen (2005), On the generation, propagation, and reflection of Alfvén waves from the solar photosphere to the distant heliosphere, Astrophys. J. Suppl. Ser., 156, 265-293.

Cranmer, S. R., W. H. Matthaeus, B. A. Breech, and J. C. Kasper (2009), Empirical constraints on proton and electron heating in the fast solar wind, Astrophys. J., 702, 1604-1614.

Dasso, S., L. J. Milano, W. H. Matthaeus, and C. W. Smith (2005) Anisotropy in fast and slow solar wind fluctuations, Astrophys. J., 635, L181-L184.

de Kármán, T., and L. Howarth (1938), On the statistical theory of isotropic turbulence, Proc. R. Soc. A, 164, 192-215.

Dobrowolny, M., A. Mangeney, and P. Veltri (1980), Fully developed anisotropic hydromagnetic turbulence in interplanetary space, Phys. Rev. Lett., 45, 144-147.

Dröge, W., Y. Y. Kartavykh, B. Klecker, and G. A. Kovaltsov (2010), Anisotropic three-dimensional focused transport of solar energetic particles in the inner heliosphere, Astrophys. J., 709, 912-919, doi:10.1088/ 0004-637X/709/2/912.

Florinski, V., and N. V. Pogorelov (2009), Four-dimensional transport of galactic cosmic rays in the outer heliosphere and heliosheath, Astrophys. J., 701, 642-651, doi:10.1088/0004-637X/701/1/642.

Florinski, V., G. P. Zank, and N. V. Pogorelov (2003), Galactic cosmic ray transport in the global heliosphere, J. Geophys. Res., 108(A6), 1228 , doi:10.1029/2002JA009695. 
Galtier, S., S. V. Nazarenko, A. C. Newell, and A. Pouquet (2000), A weak turbulence theory for incompressible magnetohydrodynamics, J. Plasma Phys., 63, 447-488.

Galtier, S., S. V. Nazarenko, A. C. Newell, and A. Pouquet (2002), Anisotropic turbulence of shear-Alfvén waves, Astrophys. J., 564, L49-L52.

Goldreich, P., and S. Sridhar (1995), Toward a theory of interstellar turbulence: II. Strong Alfvénic turbulence, Astrophys. J., 438, 763-775.

Grappin, R. (1986), Onset and decay of two-dimensional magnetohydrodynamic turbulence with velocity-magnetic field correlation, Phys. Fluids, 29, 2433-2443.

Grappin, R., U. Frisch, J. Léorat, and A. Pouquet (1982), Alfvénic fluctuations as asymptotic states of MHD turbulence, Astron. Astrophys., 105, 6-14.

Grappin, R., A. Pouquet, and J. Léorat (1983), Dependence of MHD turbulence spectra on the velocity field-magnetic field correlation, Astron. Astrophys., 126, 51-58.

Horbury, T., M. A. Forman, and S. Oughton (2005), Spacecraft observations of solar wind turbulence: An overview, Plasma Phys. Controlled Fusion, 47, B703-B717, doi:10.1088/0741-3335/47/12B/S52.

Horbury, T. S., M. Forman, and S. Oughton (2008), Anisotropic scaling of magnetohydrodynamic turbulence, Phys. Rev. Lett., 101, 175005, doi:10.1103/PhysRevLett.101.175005.

Hossain, M., P. C. Gray, D. H. Pontius Jr., W. H. Matthaeus, and S. Oughton (1995), Phenomenology for the decay of energy-containing eddies in homogeneous MHD turbulence, Phys. Fluids, 7, 2886-2904, doi: $10.1063 / 1.868665$

Iroshnikov, P. S. (1963), Turbulence of a conducting fluid in a strong magnetic field, Astron. Zh., 40, 742-750 [Reprinted in Sov. Astron., 7 566-571, 1964].

Isenberg, P. A. (2005), Turbulence-driven solar wind heating and energization of pickup protons in the outer heliosphere, Astrophys. J., 623 502-510.

Isenberg, P. A., C. W. Smith, and W. H. Matthaeus (2003), Turbulent heating of the distant solar wind by interstellar pickup protons, Astrophys. J., 592, 564-573.

Isenberg, P. A., C. W. Smith, W. H. Matthaeus, and J. D. Richardson (2010a), Turbulent heating of the distant solar wind by interstellar pickup protons in a decelerating flow, Astrophys. J., 719, 716-721, doi:10.1088/ 0004-637X/719/1/716.

Isenberg, P. A., S. Oughton, C. W. Smith, and W. H. Matthaeus (2010b), Solar wind turbulent heating by interstellar pickup protons: 2-component model, in Pickup Ions Throughout the Heliosphere and Beyond: Proceedings of the 9th Annual International Astrophysics Conference, edited by J. le Roux et al., AIP Conf. Proc., 1302, 180-185, doi:10.1063/ 1.3529967.

Joyce, C. J., C. W. Smith, P. A. Isenberg, N. Murphy, and N. A. Schwadron (2010), Excitation of low-frequency waves in the solar wind by newborn interstellar pickup ions $\mathrm{H}^{+}$and $\mathrm{He}^{+}$as seen by Voyager at $4.5 \mathrm{AU}$, Astrophys. J., 724, 1256-1261, doi:10.1088/0004-637X/724/2/1256.

King, J. H., and N. E. Papitashvili (1994), Interplanetary medium data book, suppl. 5, 1988-1993, Rep. NSSDC/WDC-A-R\&S 94-08, NASA Greenbelt, Md.

Kolmogorov, A. N. (1941), Local structure of turbulence in an incompressible viscous fluid at very high Reynolds numbers, Dokl. Akad. Nauk SSSR, 30, 301-305, [Reprinted in Proc. R. Soc. A, 434, 9-13, 1991].

Kraichnan, R. H. (1965), Inertial-range spectrum of hydromagnetic turbulence, Phys. Fluids, 8, 1385-1387.

MacBride, B. T., C. W. Smith, and B. J. Vasquez (2010), Inertial-range anisotropies in the solar wind from 0.3 to $1 \mathrm{AU}$ : Helios 1 observations, J. Geophys. Res., 115, A07105, doi:10.1029/2009JA014939.

Matthaeus, W. H., and M. L. Goldstein (1982), Measurement of the rugged invariants of magnetohydrodynamic turbulence in the solar wind, J. Geophys. Res., 87(A8), 6011-6028, doi:10.1029/JA087iA08p06011.

Matthaeus, W. H., and Y. Zhou (1989), Extended inertial range phenomenology of magnetohydrodynamic turbulence, Phys. Fluids B, 1 , 1929-1931.

Matthaeus, W. H., M. L. Goldstein, and D. A. Roberts (1990), Evidence for the presence of quasi-two-dimensional nearly incompressible fluctuations in the solar wind, J. Geophys. Res., 95(A12), 20,673-20,683, doi:10.1029/JA095iA12p20673.

Matthaeus, W. H., S. Oughton, D. H. Pontius, and Y. Zhou (1994), Evolution of energy-containing turbulent eddies in the solar wind, J. Geophys. Res., 99(A10), 19,267-19,287, doi:10.1029/94JA01233.

Matthaeus, W. H., G. P. Zank, and S. Oughton (1996), Phenomenology of hydromagnetic turbulence in a uniformly expanding medium, J. Plasma Phys., 56, 659-675, doi:10.1017/S0022377800019516.

Matthaeus, W. H., G. P. Zank, C. W. Smith, and S. Oughton (1999), Turbulence, spatial transport, and heating of the solar wind, Phys. Rev. Lett., 82, 3444-3447, doi:10.1103/PhysRevLett.82.3444.
Matthaeus, W. H., J. Minnie, B. Breech, S. Parhi, J. W. Bieber, and S. Oughton (2004), Transport of cross helicity and radial evolution of Alfvénicity in the solar wind, Geophys. Res. Lett., 31, L12803, doi:10.1029/2004GL019645.

Matthaeus, W. H., S. Dasso, J. M. Weygand, L. J. Milano, C. W. Smith, and M. G. Kivelson (2005), Spatial correlation of solar-wind turbulence from two-point measurements, Phys. Rev. Lett., 95, 231101, doi:10.1103/ PhysRevLett.95.231101.

Milano, L. J., S. Dasso, W. H. Matthaeus, and C. W. Smith (2004), Spectral distribution of the cross helicity in the solar wind, Phys. Rev. Lett., 93, 155005, doi:10.1103/PhysRevLett.93.155005.

Narita, Y., K.-H. Glassmeier, F. Sahraoui, and M. L. Goldstein (2010), Wave-vector dependence of magnetic-turbulence spectra in the solar wind, Phys. Rev. Lett., 104, 171101, doi:10.1103/PhysRevLett.104. 171101.

Ng, C. S., A. Bhattacharjee, D. Munsi, P. A. Isenberg, and C. W. Smith (2010), Kolmogorov versus Iroshnikov-Kraichnan spectra: Consequences for ion heating in the solar wind, J. Geophys. Res., 115 A02101, doi:10.1029/2009JA014377.

Osman, K. T., and T. S. Horbury (2009), Quantitative estimates of the slab and 2-D power in solar wind turbulence using multispacecraft data J. Geophys. Res., 114, A06103, doi:10.1029/2008JA014036.

Oughton, S., E. R. Priest, and W. H. Matthaeus (1994), The influence of a mean magnetic field on three-dimensional MHD turbulence, J. Fluid Mech., 280, 95-117, doi:10.1017/S0022112094002867.

Oughton, S., P. Dmitruk, and W. H. Matthaeus (2006), A two-component phenomenology for homogeneous magnetohydrodynamic turbulence, Phys. Plasmas, 13, 042306, doi:10.1063/1.2188088.

Parker, E. N. (1965), Dynamical theory of the solar wind, Space Sci. Rev., 4, 666-708, doi:10.1007/BF00216273.

Pearson, B. R., T. A. Yousef, N. E. L. Haugen, A. Brandenburg, and P.-A Krogstad (2004), Delayed correlation between turbulent energy injection and dissipation, Phys. Rev. E, 70, 056301, doi:10.1103/PhysRevE.70. 056301 .

Pei, C., J. W. Bieber, B. Breech, R. A. Burger, J. Clem, and W. H. Matthaeus (2010), Cosmic ray diffusion tensor throughout the heliosphere, J. Geophys. Res., 115, A03103, doi:10.1029/2009JA014705.

Perri, S., and A. Balogh (2010), Differences in solar wind cross-helicity and residual energy during the last two solar minima, Geophys. Res. Lett., 37, L17102, doi:10.1029/2010GL044570.

Podesta, J. J. (2009), Dependence of solar-wind power spectra on the direction of the local mean magnetic field, Astrophys. J., 698, 986-999, doi:10.1088/0004-637X/698/2/986.

Richardson, J. D., and C. W. Smith (2003), The radial temperature profile of the solar wind, Geophys. Res. Lett., 30(5), 1206, doi:10.1029/ 2002GL016551.

Roberts, D. A., L. W. Klein, M. L. Goldstein, and W. H. Matthaeus (1987a), The nature and evolution of magnetohydrodynamic fluctuations in the solar wind: Voyager observations, J. Geophys. Res., 92(A10), 11,021-11,040, doi:10.1029/JA092iA10p11021.

Roberts, D. A., M. L. Goldstein, L. W. Klein, and W. H. Matthaeus (1987b), Origin and evolution of fluctuations in the solar wind: Helios observations and Helios-Voyager comparisons, J. Geophys. Res., 92(A11), 12,023-12,035, doi:10.1029/JA092iA11p12023.

Shebalin, J. V., W. H. Matthaeus, and D. Montgomery (1983), Anisotropy in MHD turbulence due to a mean magnetic field, J. Plasma Phys., 29, $525-547$.

Smith, C. W. (2003), The geometry of turbulent magnetic fluctuations at high heliographic latitude, in Solar Wind Ten, vol. 679, edited by M. Velli, R. Bruno, and F. Malara, pp. 413-416, Am. Inst. of Phys., Melville, N. Y.

Smith, C. W., W. H. Matthaeus, G. P. Zank, N. F. Ness, S. Oughton, and J. D. Richardson (2001), Heating of the low-latitude solar wind by dissipation of turbulent magnetic fluctuations, J. Geophys. Res., 106(A5), 8253-8272, doi:10.1029/2000JA000366

Smith, C. W., P. A. Isenberg, W. H. Matthaeus, and J. D. Richardson (2006), Turbulent heating of the solar wind by newborn interstellar pickup protons, Astrophys. J., 638, 508-517.

Sreenivasan, K. R. (1995), On the universality of the Kolmogorov constant, Phys. Fluids, 7, 2778-2784.

Taylor, G. I. (1938), The spectrum of turbulence, Proc. R. Soc. A, 164 476-490

Tu, C.-Y., and E. Marsch (1993), A model of solar wind fluctuations with two components: Alfvén waves and convective structures, J. Geophys. Res., 98(A2), 1257-1276, doi:10.1029/92JA01947.

Tu, C.-Y., and E. Marsch (1995), MHD Structures, Waves and Turbulence in the Solar Wind, Kluwer, Dordrecht, Netherlands.

Usmanov, A. V., and M. L. Goldstein (2010), Alfvénic turbulence simulation in a realistic solar wind, in Numerical Modeling of Space Plasma 
Flow: ASTRONUM-2009, Astron. Soc. Pac. Conf. Ser., vol. 429, edited by N. V. Pogorelov, E. Audit, and G. P. Zank, pp. 51-58, Astron. Soc. of the Pac., San Francisco, Calif.

Weygand, J. M., W. H. Matthaeus, S. Dasso, M. G. Kivelson, L. M. Kistler, and C. Mouikis (2009), Anisotropy of the Taylor scale and the correlation scale in plasma sheet and solar wind magnetic field fluctuations, J. Geophys. Res., 114, A07213, doi:10.1029/2008JA013766.

Wicks, R. T., M. J. Owens, and T. S. Horbury (2010), The variation of solar wind correlation lengths over three solar cycles, Sol. Phys., 262, 191-198, doi:10.1007/s11207-010-9509-4.

Williams, L. L., and G. P. Zank (1994), Effect of magnetic field geometry on the wave signature of the pickup of interstellar neutrals, J. Geophys. Res., 99(A10), 19,229-19,244, doi:10.1029/94JA01657.

Williams, L. L., G. P. Zank, and W. H. Matthaeus (1995), Dissipation of pickup-induced waves: A solar wind temperature increase in the outer heliosphere?, J. Geophys. Res., 100(A9), 17,059-17,067, doi:10.1029/ 95JA01261.

Yokoi, N., and F. Hamba (2007), An application of the turbulent magnetohydrodynamic residual-energy equation model to the solar wind, Phys. Plasmas, 14, 112904, doi:10.1063/1.2792337.

Zank, G. P., and W. H. Matthaeus (1992a), The equations of reduced magnetohydrodynamics, J. Plasma Phys., 48, 85-100.

Zank, G. P., and W. H. Matthaeus (1992b), Waves and turbulence in the solar wind, J. Geophys. Res., 97(A11), 17,189-17,194, doi:10.1029/ 92JA01734.
Zank, G. P., and W. H. Matthaeus (1993), Nearly incompressible fluids. II: Magnetohydrodynamics, turbulence, and waves, Phys. Fluids A, 5, 257-273.

Zank, G. P., W. H. Matthaeus, and C. W. Smith (1996), Evolution of turbulent magnetic fluctuation power with heliocentric distance, J. Geophys. Res., 101, 17,093-17,107.

Zank, G. P., W. H. Matthaeus, J. W. Bieber, and H. Moraal (1998), The radial and latitudinal dependence of the cosmic ray diffusion tensor in the heliosphere, J. Geophys. Res., 103(A2), 2085-2097, doi:10.1029/ 97JA03013.

Zhou, Y., and W. H. Matthaeus (1990), Transport and turbulence modeling of solar wind fluctuations, J. Geophys. Res., 95(A7), 10,291-10,311, doi:10.1029/JA095iA07p10291.

Zhou, Y., W. H. Matthaeus, and P. Dmitruk (2004), Magnetohydrodynamic turbulence and time scales in astrophysical and space plasmas, Rev. Mod. Phys., 76, 1015-1035.

B. Breech, U. S. Army Research Laboratory, Aberdeen Proving Grounds, Aberdeen, MD 21005, USA

P. A. Isenberg and C. W. Smith, Institute for the Study of Earth, Oceans, and Space, University of New Hampshire, Durham, NH 03824, USA.

W. H. Matthaeus, Bartol Research Institute, 217 Sharp Lab, University of Delaware, Newark, DE 19716, USA. (whm@udel.edu)

S. Oughton, Department of Mathematics, University of Waikato, Private Bag 3105, Hamilton 3240, New Zealand. 